Supporting Information for:

\title{
Control of the Redox Activity of PbS Quantum Dots by Tuning Electrostatic Interactions at the Quantum Dot/Solvent Interface
}

Chen He, David J. Weinberg, Alexander B. Nepomnyashchii, Shichen Lian, and Emily A. Weiss*

Department of Chemistry, Northwestern University, 2145 Sheridan Rd., Evanston, IL 60208 *corresponding author. Email: e-weiss@northwestern.edu

Synthetic Procedures for Oleate-capped PbS QDs. We synthesized $1.6 \mathrm{~nm}$ oleate-capped $\mathrm{PbS}$ QDs using a procedure adapted from that of Hines and Scholes. ${ }^{1} \mathrm{We}$ mixed $0.36 \mathrm{~g} \mathrm{PbO}$ and $2.0 \mathrm{~mL}$ oleic acid (OA) with $18.0 \mathrm{~mL}$ 1-octadecene (ODE) in a 50-mL three-neck round bottom flask at room temperature. Heating the mixture up to $150{ }^{\circ} \mathrm{C}$ with constant stirring under $\mathrm{N}_{2}$ flow for an hour produced a clear and colorless solution. We cooled the mixture to $110^{\circ} \mathrm{C}$, and injected $0.17 \mathrm{~mL}$ of hexamethyldisilathiane dissolved in $8 \mathrm{~mL}$ of ODE. The solution turned from orange to brown within 3 seconds. After 10 minutes, we used an ice bath to cool the reaction mixture to room temperature. The product was separated into four $50-\mathrm{mL}$ centrifuge tubes for further purification, as is described in the main text. We purified the QDs by first washing the reaction mixture with acetone (6:1 by volume), and centrifuging it at $3500 \mathrm{rpm}$ for $20 \mathrm{~min}$. We then decanted the supernatant, dried the QD pellet, redispersed the QDs in $5 \mathrm{~mL}$ hexanes, and precipitated the QDs two additional times, as described above, using $12.5 \mathrm{~mL}$ methanol and acetone, respectively, as the non-solvents. The cleaned $\mathrm{PbS}$ QDs were finally dispersed in a minimal amount of hexanes to form the stock solution.

\section{Sizing of PbS QDs via UV-vis Ground State Absorption and Transmission Electron} Microscopy. All ground state absorption spectra of solutions of oleate-capped PbS QDs were obtained on a Varian Cary 5000 spectrometer using a $2 \mathrm{~mm} / 10 \mathrm{~mm}$ dual pathlength quartz cuvette (in which we excite along the $2 \mathrm{~mm}$ axis). We corrected the baselines of all spectra with hexanes prior to measurement, and determined the size of the synthesized PbS QDs (and their respective extinction coefficient) from the position of the first excitonic peak $(\sim 985 \mathrm{~nm})$ using the calibration curve published by Moreels et al. ${ }^{2}$ All concentrations of QDs were calculated from the absorbance of QDs at $400 \mathrm{~nm}$ (Figure S1A). In order to verify the accuracy of this technique, we performed 

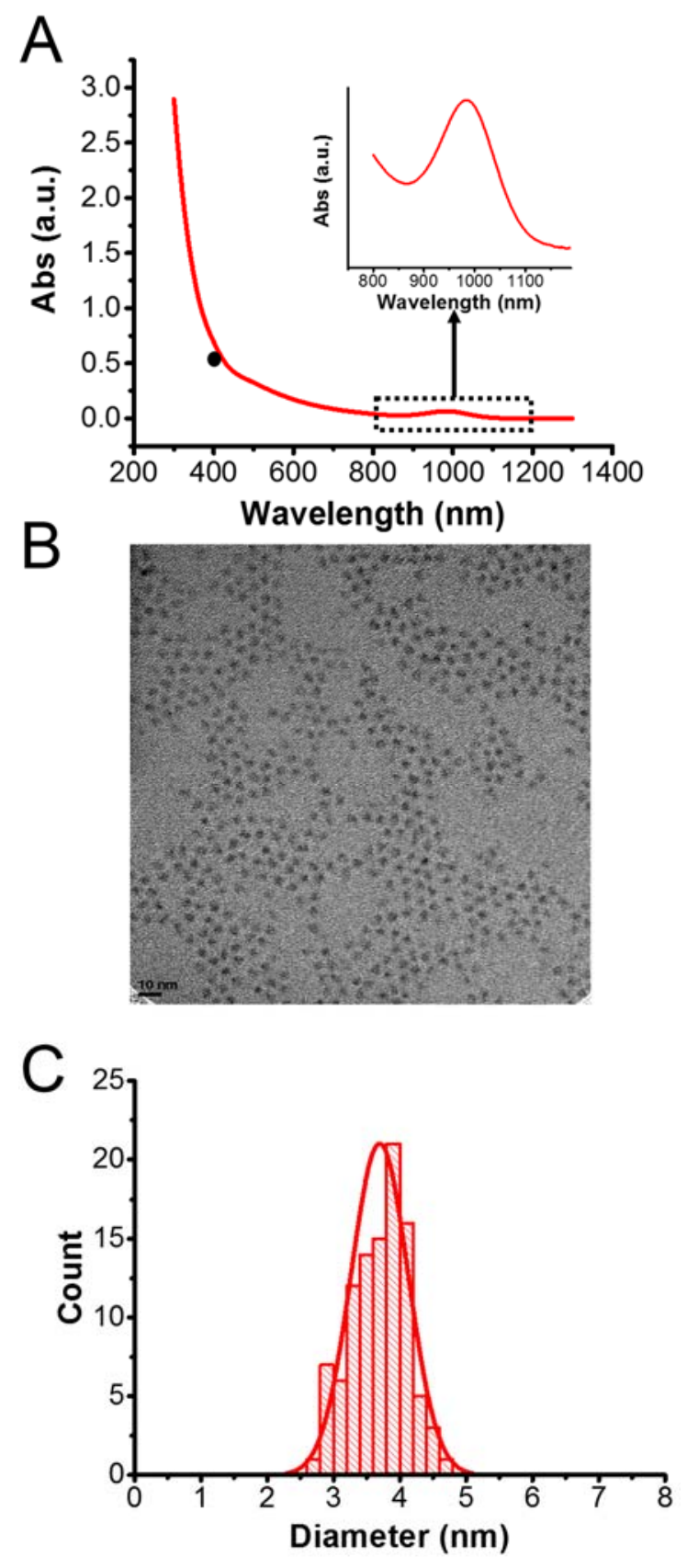

Figure S1. A) UV-Vis ground state absorption spectrum (300 nm-1300 nm) of $4.5 \mu \mathrm{M}$ oleatecapped $\mathrm{PbS}$ QDs (1.6 nm radius) in hexanes. Inset: the same spectrum zoomed at the first excitonic peak (985 $\mathrm{nm})$. The absorbance used for concentration calibration is highlighted. B) Representative transmission electron micrograph (TEM) image of oleate-capped PbS QDs. C) Histogram of nanocrystal diameter constructed from 101 QD diameter measurements in Image J. The average and standard deviation for the radius of these particles are 1.8 and $0.2 \mathrm{~nm}$, respectively. 
transmission electron microscopy experiments using a JEOL JEM-2100F FAST TEM. We prepared TEM samples by drop-casting a $\sim 12 \mu \mathrm{M}$ solution of PbS QDs in hexanes onto a Carbon Type B film (Ted Pella, Inc), and Figure S1B shows a representative TEM image of these QDs. We analyzed $101 \mathrm{PbS}$ QDs using the Image $^{3}$ software package, and determined that the average diameter of these particles is $3.6 \pm 0.4 \mathrm{~nm}$, Figure $\mathbf{S 1 C}$. The $1.6 \mathrm{~nm}$ radius for oleate-capped QDs that we predicted from UV-Vis spectroscopy is within the error of our TEM measurement.

Cyclic Voltammetric (CV) and Ground State Absorption Study of 9,10-Anthraquinone2-Sulfonic Acid Sodium Salt (AQ). In order to determine the redox potential of AQ, we first performed cyclic voltammetry experiments using a three-electrode cell with a 3-mm radius glassy carbon electrode as the working electrode, saturated $\mathrm{Ag} / \mathrm{AgCl}$ as the reference electrode, and platinum wire as the counter electrode. Measurements were taken with Princeton applied research potentiostats using $0.5 \mathrm{mM}$ deaerated solutions of $\mathrm{AQ}$, with $0.25 \mathrm{M} \mathrm{NaNO}_{3}$ added as the supporting electrolyte. The $\mathrm{pH}$ of the solution is adjusted to 10.0 using $\mathrm{NaOH}$ to mimic the actual condition for PL quenching experiments $(\mathrm{pH}=9.6 \sim 10.3)$.

Cyclic voltammogram in Figure S2A shows quasi-reversible reduction of AQ under various scanning rates $(0.05 \mathrm{~V} / \mathrm{s}, 0.1 \mathrm{~V} / \mathrm{s}, 0.25 \mathrm{~V} / \mathrm{s}$ and $0.5 \mathrm{~V} / \mathrm{s})$. The observed reduction potential is -0.59 $\mathrm{V}$ vs. $\mathrm{Ag} / \mathrm{AgCl}$ reference electrode, which, after correction using eq $\mathbf{S 1}^{4}$, corresponds to $\sim-4.1 \mathrm{eV}$ for the LUMO energy of AQ relative to vacuum.

$$
\begin{gathered}
E(\text { vs. vacuum })=-E(\text { vs. NHE })-4.43 \mathrm{eV} \\
=-[E(\text { vs. saturated } \mathrm{Ag} / \mathrm{AgCl})+0.199 \mathrm{eV}]-4.43 \mathrm{eV} \\
=-\left[E_{\text {reduction peak potential }}+0.0285 \mathrm{eV}+0.199 \mathrm{eV}\right]-4.43 \mathrm{eV}
\end{gathered}
$$

$\mathrm{UV}-\mathrm{Vis}$ ground state absorption spectrum of AQ (collected at a concentration of $260 \mu \mathrm{M}$ ), Figure S2B, shows that the optical transition with the minimal energy is centered at $\sim 330 \mathrm{~nm}$, which translates into a $3.8 \mathrm{eV}$ excitation energy between the HOMO and the LUMO. Therefore, we subtract this energy from the LUMO energy that we measured using $\mathrm{CV}$, and determine that the HOMO energy of AQ is $\sim-7.9 \mathrm{eV}$, see Figure $\mathbf{1 C}$ in the main text.

Ground state absorption spectra show that up to $15 \%$ of PbS QDs are lost during the preparation of MHA/MHO-capped PbS QDs. We used UV-Vis spectrophotometer to determine the concentration of PbS QDs by the amplitude of their ground state absorbance at $400 \mathrm{~nm}^{2}$ Then we compared the total amount of PbS QDs present in solution before and after ligand exchange, 
and calculated the percentage of PbS QDs lost, as listed in Table S1, to be between 14.4\% and $0.0 \%$ for samples $1-6$.

All of the native oleate ligands are displaced upon adding 400 eq. of thiols to PbS QDs during the ligand exchange. Figure S3A shows the full spectra of MHA/MHO-capped watersoluble PbS QDs $(13.2 \mu \mathrm{M})$, with six different compositions in their ligand shells. Figure S3B compares the representative NMR spectrum of water-soluble QDs (after exchange) with oleatecapped QDs (before ligand exchange). For QDs that are dispersed in aqueous solution, the absence of signal from bound oleate (broad feature centered at $5.69 \mathrm{ppm}$ ) indicates that all the native oleate ligands are completely displaced upon adding 400 eq. of thiols.

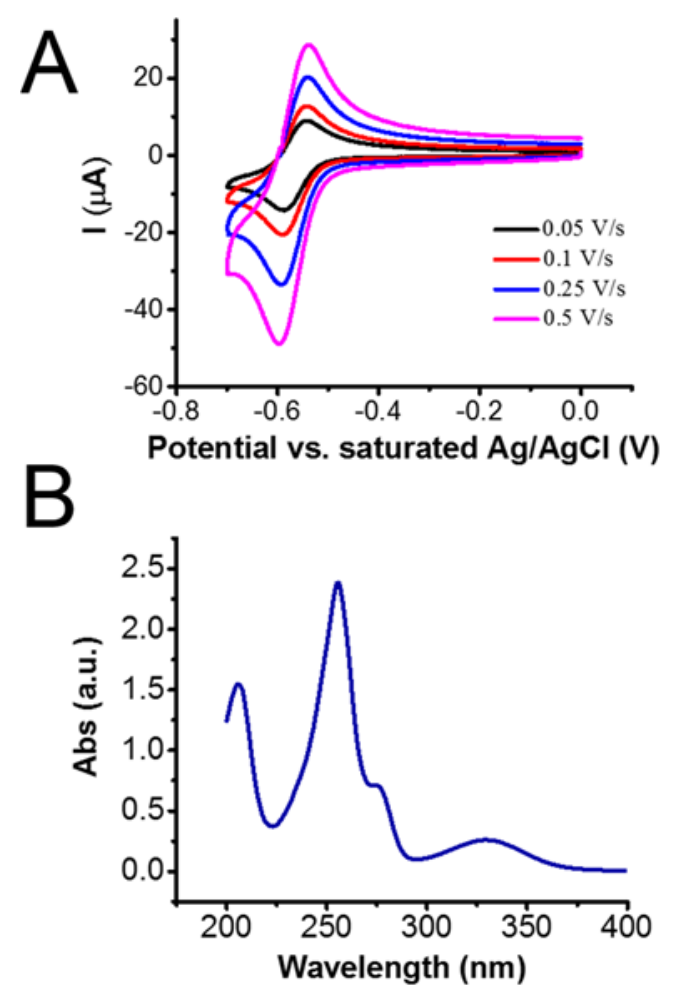

Figure S2. A) Cyclic voltammogram for AQ under multiple scanning rates. B) UV-Vis ground state absorption spectrum of AQ $(260 \mu \mathrm{M})$.

Table S1. Percentage of PbS QDs Lost during the Ligand Exchange for Sample 1-6.

\begin{tabular}{ccc}
\hline No. of samples & Eq. of MHA/MHO added & Percentage of PbS QDs lost \\
\hline 1 & $400 / 0$ & 9.4 \\
2 & $320 / 80$ & 14.4 \\
3 & $280 / 120$ & 13.4 \\
4 & $240 / 160$ & 8.4 \\
5 & $160 / 240$ & 9.3 \\
6 & $80 / 320$ & 0.0 \\
\hline
\end{tabular}




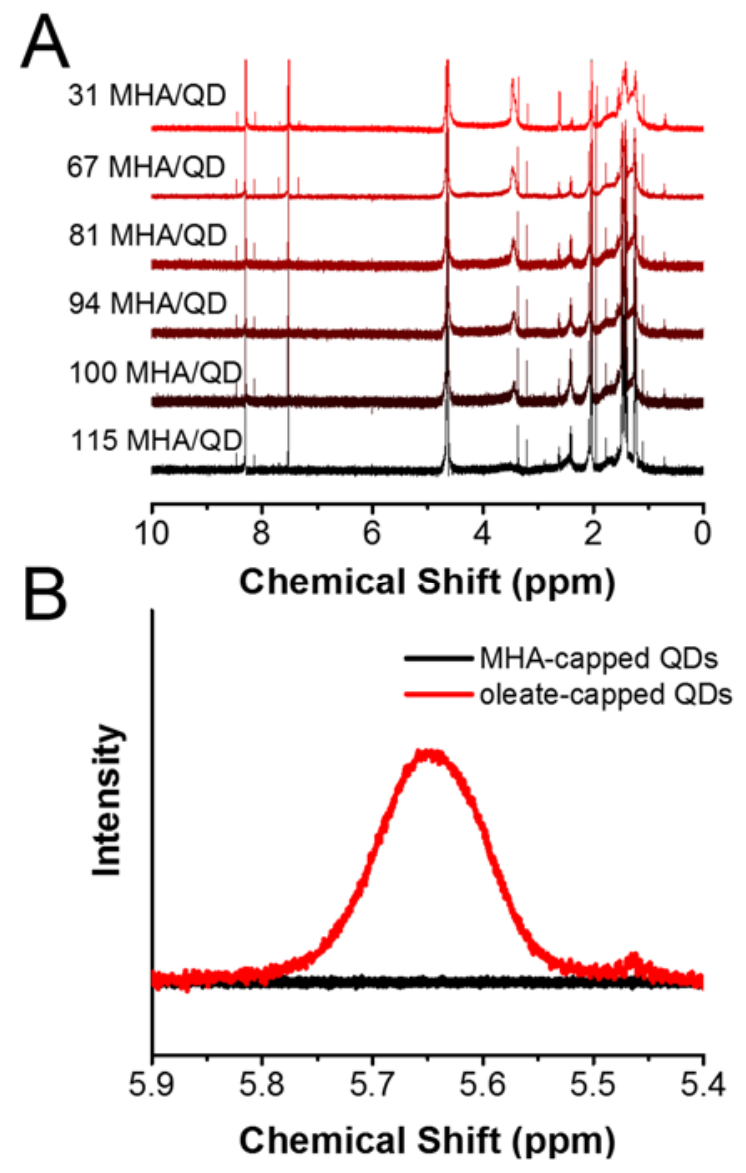

Figure S3. A) Full ${ }^{1} \mathrm{H}$ NMR spectra for Samples 1-6 in $\mathrm{D}_{2} \mathrm{O}$. The sharp singlets at 8.33 ppm, $7.53 \mathrm{ppm}$, and $4.65 \mathrm{ppm}$ correspond to sodium formate (added as an internal standard for integration, see next section), chloroform residue, and $\mathrm{H}_{2} \mathrm{O}$ impurity, respectively. B) ${ }^{1} \mathrm{H} \mathrm{NMR}$ spectra for 13.2 $\mu \mathrm{M}$ MHA- and oleate-capped PbS QDs.

Table S2. The Number of Bound and Free Oleate (OA) per QD and the Total Number of Binding Sites on the Surface of PbS QDs.

\begin{tabular}{ccccc}
\hline $\begin{array}{c}\text { Eq. of HT added } \\
\text { to PbS QDs }\end{array}$ & $\begin{array}{c}\text { No. of Free } \\
\text { OA/QD }\end{array}$ & $\begin{array}{c}\text { No. of Bound } \\
\text { OA/QD }\end{array}$ & $\begin{array}{c}\text { Total No. of } \\
\text { OA/QD }^{b}\end{array}$ & $\begin{array}{c}\text { Total No. of } \\
\text { binding sites }^{c}\end{array}$ \\
\hline 0 HT & 10 & $200-220$ & $210-230$ & $200-220$ \\
200 HT & 140 & 80 & 220 & 210 \\
400 HT & 160 & 40 & 200 & 190 \\
800 HT & $180-200$ & 20 & $200-220$ & $190-210$ \\
\hline
\end{tabular}

${ }^{a}$ Calculated by integrating the signals of OA vinyl protons in ${ }^{1} \mathrm{H}$ NMR spectra as described in the text.

${ }^{b}$ Estimated as the sum of the number of free and bound OA per QD.

${ }^{c}$ Calculated by subtracting the number of free OA (with no HT added) from the total number of OA per QD estimated from $b$. 


\section{Quantification of MHA and MHO within the Mixed Monolayer Ligand Shell of Water-}

soluble PbS QDs. Figure S4 shows the signals from vinyl protons of bound oleate (5.69 ppm, broad) and free oleate species (5.50 ppm, sharp multiplet) in the ${ }^{1} \mathrm{H}$ NMR spectrum of oleatecoated QDs treated with different concentrations of hexanethiol (HT). As the amount of added HT increases, the signal from the vinyl protons of bound oleate decreases, and the signal from the vinyl protons for freely-diffusing oleate species increases. The sum of the integrated signals corresponding to bound and free populations of oleate species (measured relative to an internal biphenyl standard with a signal at $7.45 \mathrm{ppm}$ ) is between 200 and 230 ligands per QD at all points in the titration, see Table S2 (the errors arise from the discrepancy between integrating the whole peak vs. $2 \times$ integrating half the peak). We therefore estimate the maximum number of bound oleate per PbS QD (which equals the total number of oleate per QD minus the number of free oleate with zero HT added) to be 190 - 220. This number is used to approximate the number of bound MHO per QD, as we described in Table 1 of the main text.

We acquired ${ }^{1} \mathrm{H}$ NMR spectra for all the six water-soluble PbS QD samples $(13.2 \mu \mathrm{M})$ after ligand exchange and concentration calibration, as we described in the previous section, and used a sum of five Lorentzian functions to fit and quantify the bound (one broad peak centered at $\sim 2.07$ ppm) and free (sharp triplet centered at $\sim 2.03 \mathrm{ppm}$ ) MHA species as well as the singlet impurity located at $\sim 2.08 \mathrm{ppm}$. The respective ${ }^{1} \mathrm{H}$ NMR spectra for Sample 1-6 (zoomed in at 1.96-2.20 ppm) are shown in Figure S5. The same spectra zoomed in at 3.30-3.70 ppm, the chemical shift of methylene protons alpha to the hydroxyl group in MHO, are also shown in Figure S6. We only observe a broad feature of MHO for all QD samples, with no clear distinction between bound and free populations, which suggests that either all the MHO ligands are bound to the surface of QD, or there's a fast exchange between bound and free populations of MHO. ${ }^{5}$ The poor resolution of spectra also made it difficult to integrate these peaks accurately. We therefore chose to approximate the upper limit of the number of bound MHO per QD using the technique described in the main text. 


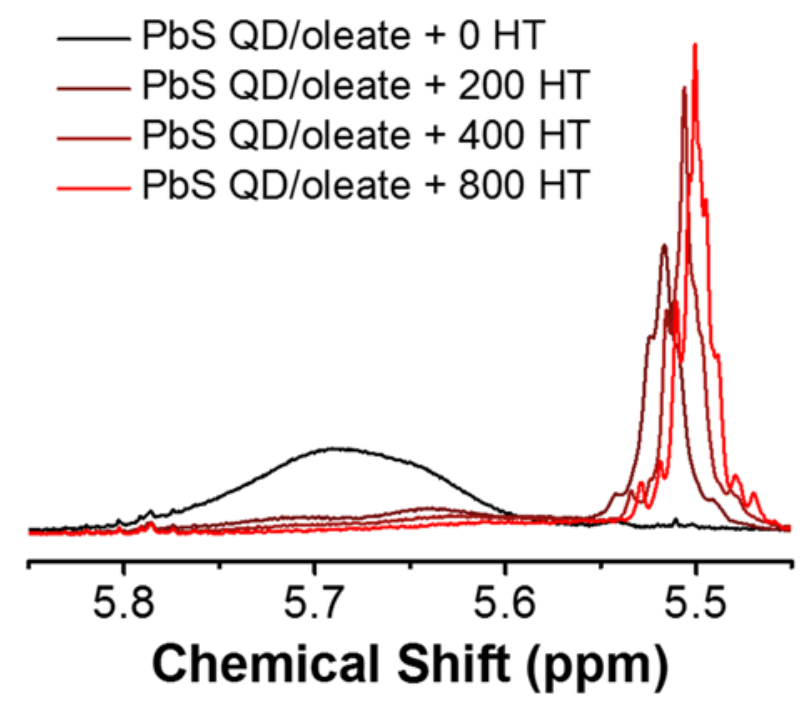

Figure S4. Titration of oleate-capped PbS QDs $(\mathrm{R}=1.6 \mathrm{~nm})$ with $0,200,400$, and 800 equivalents of hexanethiol (HT) per QD. The broad feature at $5.69 \mathrm{ppm}$, corresponding to the vinyl protons of bound oleate, decreases in intensity, and the sharp multiplet at $\sim 5.50 \mathrm{ppm}$, corresponding to the vinyl protons of displaced oleate species, increases in intensity as more HT is added to the QDs. The number of displaced OA saturates at 180-200. Approximately 20 tightly bound oleate ligands remain bound to the QD, even upon addition of 800 eq of HT.
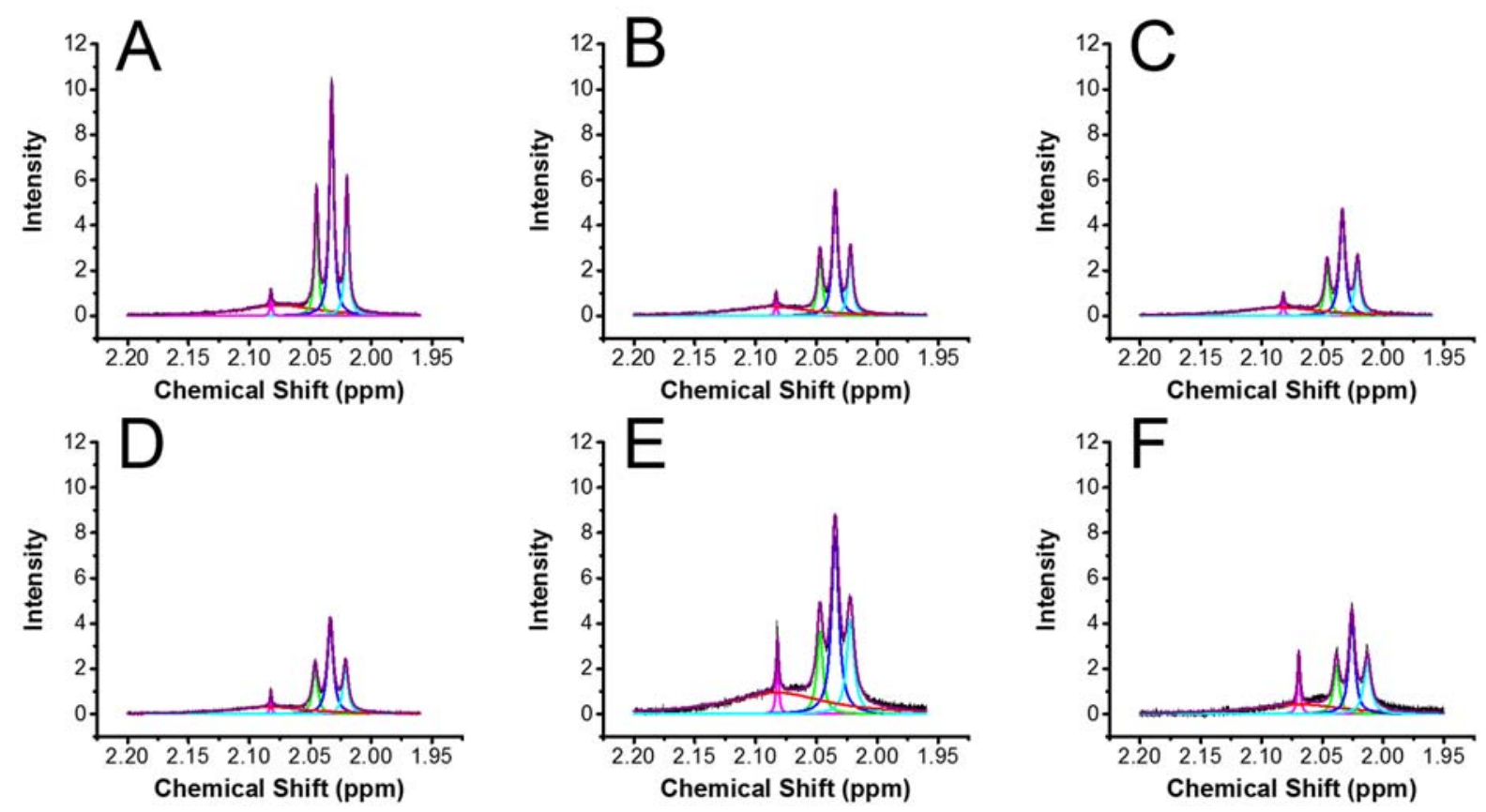

Figure S5. ${ }^{1} \mathrm{H}$ NMR spectra (zoomed in at 1.96-2.20 ppm) for Sample A) 1, B) 2, C) 3, D) 4, E) 5 and F) 6 fit by a sum of five Lorentzian functions, as we described in the main text. 

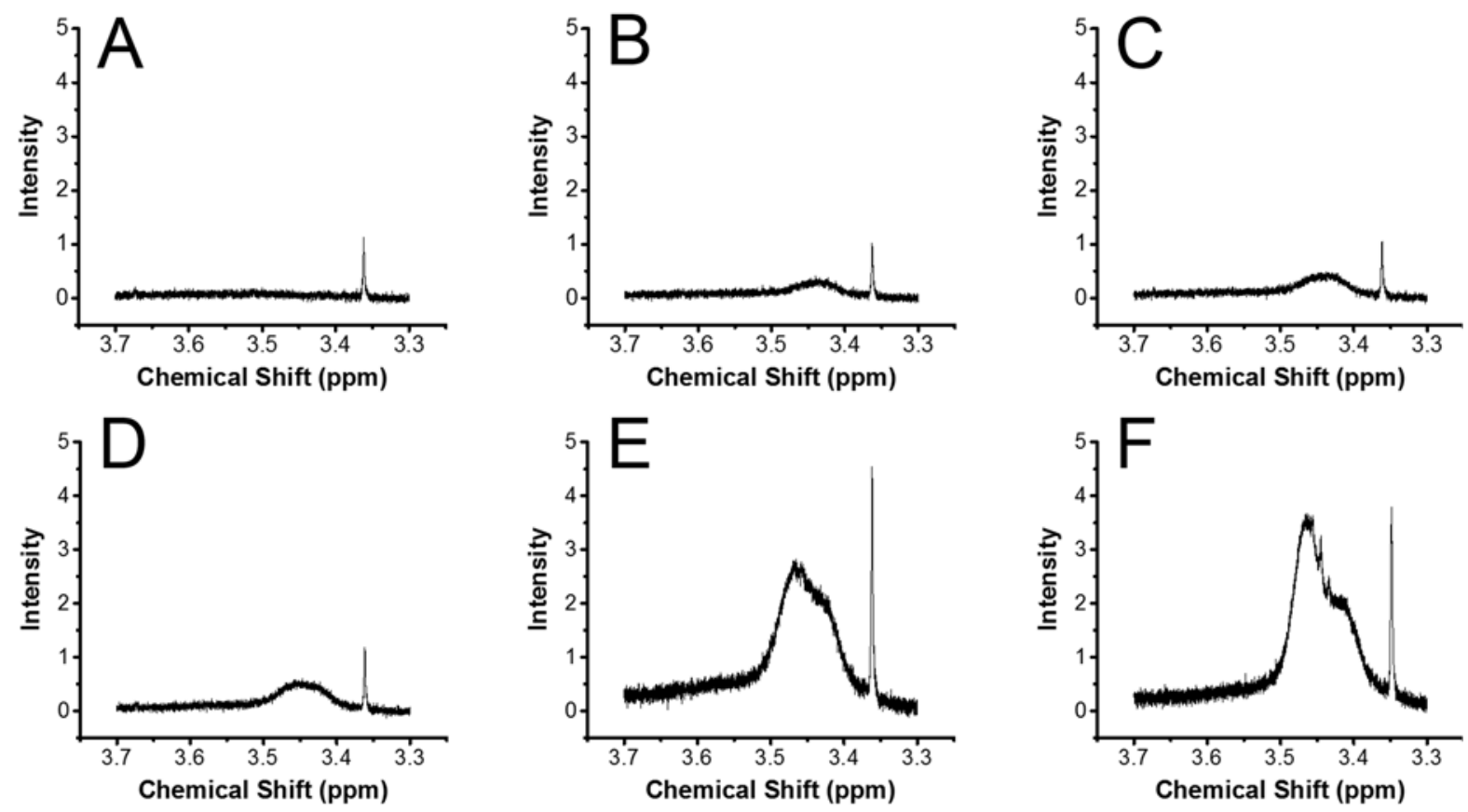

Figure S6. 1H NMR spectra (zoomed in at 3.30-3.70 ppm) for Sample A) 1, B) 2, C) 3, D) 4, E) 5 and F) 6. The sharp singlet at $3.35 \mathrm{ppm}$ originates from an unknown impurity in MHA ligands.

$\mathrm{AQ}$ is stable in aqueous solutions with the presence of water-soluble $\mathrm{PbS}$ QDs. We prepared four samples of $2.63 \mu \mathrm{M}$ MHA-capped PbS QDs with 6000 equivalents of AQ/QD added. All the samples were stored under the same protection of $\mathrm{N}_{2}$ with different treatments of light described in Figure S7. ${ }^{1} \mathrm{H}-\mathrm{NMR}$ spectra show that: (i) there's no spontaneous reaction between MHA-capped PbS QDs and AQ in the dark, (ii) when exposed to light, PbS QDs and AQ undergo a photochemical redox reaction and create a long-living paramagnetic AQ radical anion ${ }^{6}$, which could be inferred from the broadened peaks in NMR spectra, (iii) The broad features of AQ radical anion resharpen when the sample we expose to light beforehand is put back in the dark overnight, which may indicate a charge-recombination process between AQ radical anions and oxidized QDs, and (iv) we observed no new peaks in NMR spectra for any of these samples. We conclude that there's no spontaneous reaction between AQ and water-soluble PbS QDs without light, and we therefore chose to store all the samples in the dark for four hours to equilibrate before taking PL measurements in order to eliminate photochemical reactions that can be driven by room light. 


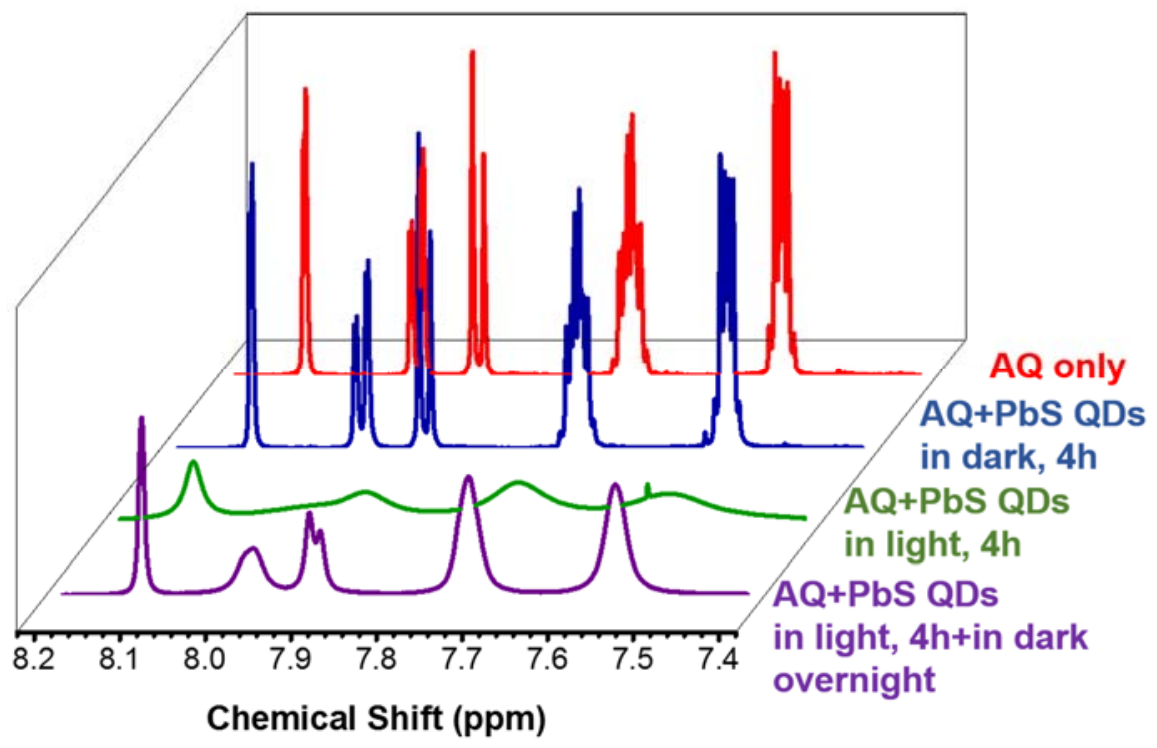

Figure S7. ${ }^{1} \mathrm{H}$ NMR spectra of AQ (red), AQ added to PbS QDs and kept in darkness for $4 \mathrm{~h}$ (blue), AQ added to PbS QDs and kept in light for $4 \mathrm{~h}$ (green), and AQ added to PbS QDs, exposed to light for $4 \mathrm{~h}$, and then kept in the dark overnight (violet).

PbS QD-AQ complexes could reach equilibrium of adsorption in four hours. We performed a time-dependent PL study on a separately prepared sample, using the same set-up described in last section (2.63 $\mu \mathrm{M}$ MHA-capped PbS QDs with 6000 equivalents of AQ/QD added). We took 17 measurements consecutively with a 30-min interval, and our result shows that the integrated PL intensity saturates in four hours after we add AQ to the QD sample, which indicates that the adsorption of $\mathrm{AQ}$ on the surface of $\mathrm{QD}$ has reached equilibrium.

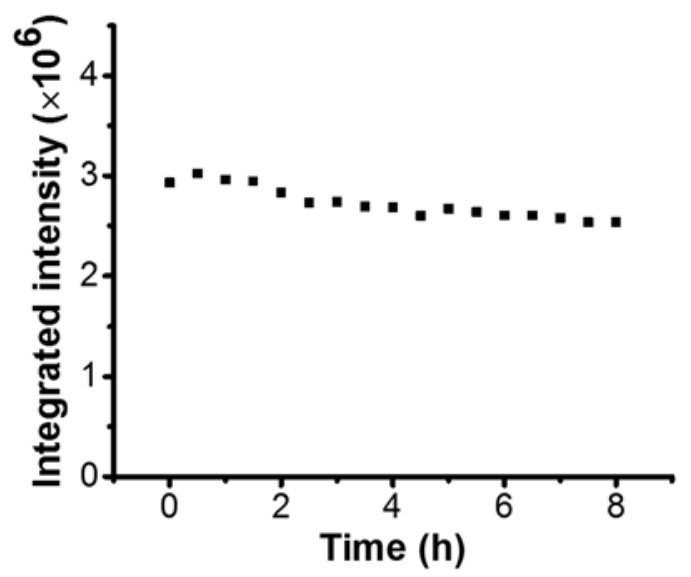

Figure S8. Time-dependent PL study for $2.63 \mu \mathrm{M}$ MHA-capped PbS with 6000 eq. of AQ/QD added as molecular acceptors. 
Transient Absorption Spectra as A Proof for Electron Transfer and Extraction of Globally-shared Intrinsic eT Rate for Each eT-active PbS QD-AQ Pair. Ultrafast Transient Absorption Spectroscopy. We split the 2.5-mJ output of a commercial amplified Ti-sapphire laser (Solstice, $1 \mathrm{kHz}, 100 \mathrm{fs}$, Spectra Physics), and guided 95\% to an optical parametric amplifier (TOPAS-C, Light Conversion) used to produce the pump wavelength $(850 \mathrm{~nm})$ for sample excitation, and 5\% to a commercial TA spectrometer (HELIOS, Ultrafast Systems) for use as the probe for TA experiments with pump-probe delay times up to $3200 \mathrm{ps.} \mathrm{Within} \mathrm{the} \mathrm{spectrometer,} \mathrm{a}$ single filament broadband continuum of probe wavelengths from 900-1400 nm was generated in a $1.2 \mathrm{~cm}$ thick sapphire plate and then passed through a long-wave pass filter to isolate nearinfrared wavelengths above $850 \mathrm{~nm}$. The probe light was then split into sample and reference beams. We combined the sample beam with the pump light at the sample, which was contained in a 2-mm quartz cuvette. The pump spot size was expanded to at least twice the size of the probe spot to compensate for any imperfections in translation stage alignment. The transmitted probe signal was collected into an optical fiber and dispersed onto an array detector. Dividing the signal from the sample beam by the signal from the reference beam allowed us to divide out any fluctuations in the probe beam intensity during the experiment. The output differential absorption spectrum $(\triangle \mathrm{A})$ was obtained through active background subtraction of the ground state spectrum by chopping the pump at $500 \mathrm{~Hz}$. The pump light was depolarized to prevent unintentional photoselection so that measurements reflect only population dynamics, and we adjusted the power to $100 \mu \mathrm{W}$ to ensure an expectation value $(<\mathrm{N}>)$ of $\sim 0.2$ in the first excitonic state of the QDs and avoid any multi-exciton effects. The solution was stirred with a magnetic stir bar to minimize local heating.

Microsecond Transient Absorption Spectroscopy. We used a commercial spectrometer (EOS, Ultrafast Systems) to collect TA spectra for pump-probe delay times from $0.5 \mathrm{~ns}-25 \mu \mathrm{s}$. The excitation beam was generated by the same method and followed the same beam path as the excitation used for the picosecond TA experiment described above. The proprietary EOS light source generates a super-continuum (400-1700 nm) probe pulse by focusing a diode laser into a photonic crystal fiber. The repetition rate of the probe pulse is $2 \mathrm{kHz}$, which is twice the repetition rate of the pump pulse, and it is triggered in sync with the pump pulse. The pump-probe delay is electronically generated and measured by an electronic timer-counter-analyzer (Pendulum). After the probe pulse passes through an 850 -nm long pass filter, it is split by a beam-splitter into sample 
and reference beams. The sample beam was sent onto the sample, which was contained in a 2-mm quartz cuvette, and focused to a spot size of $\sim 200 \mu \mathrm{m}$. The reference and sample beams are then collected in optical fibers and dispersed onto array detectors. In order to achieve reasonable signal to noise, the incident power of pump light was adjusted to be $2 \mathrm{~mW}$. The solution was stirred with a magnetic stir bar to minimize local heating.

We performed transient absorption experiments, as described above, on a set of separately prepared 6.58 $\mu \mathrm{M}$ PbS QDs with two different surface charge densities and various amounts of AQ added, which we listed in Table S3A and S3B. The samples were allowed to equilibrate in dark for four hours before we took measurements. We applied a sum of exponentials convoluted with an instrument response function (in this case, a Gaussian pulse) to fit the kinetic trace of the ground state bleach and determine the life time and relaxation dynamics of photo-excited electrons and holes on both the ultrafast and the microsecond timescale, eq S2:

$$
y=I R F \otimes \sum_{1}^{n} A_{n} e^{\frac{-\left(t-t_{0}\right)}{\tau_{n}}}
$$

Here we choose $n$ to be the minimal integer that can make the fitting converge. Figure S9 shows the microsecond TA kinetic traces of 115 MHA-capped and 31 MHA-capped PbS QDs extracted at $1014 \mathrm{~nm}$ and $1039 \mathrm{~nm}$, respectively, while the ultrafast TA kinetics are described in Figure 3, main text. The fitting parameters of these kinetic traces are listed in Table S3A and S3B. 
Table S3A. Time Constants for Decay of the QD Exciton for QD-AQ Complexes on Ultrafast Time Scale.

\begin{tabular}{|c|c|c|c|c|c|c|}
\hline $\begin{array}{l}\text { No. of } \\
\text { Sample }\end{array}$ & $\begin{array}{c}\text { No. of } \\
\text { bound MHA } \\
\text { ligands per } \\
\text { QD }\end{array}$ & $\begin{array}{l}\text { Eq. of AQ } \\
\text { bound/QDD }\end{array}$ & $P L / P L_{0}$ & $\begin{array}{c}\tau_{1}=\tau_{\mathrm{eT}} \\
(\mathrm{ps}) \\
\left(\mathrm{A}_{3}\right)^{b, c}\end{array}$ & $\begin{array}{l}\tau_{2}(\mathrm{ps}) \\
\left(\mathrm{A}_{2}\right)^{b, d}\end{array}$ & $\begin{array}{l}\tau_{3}(\mu \mathrm{s}) \\
\left(\mathrm{A}_{1}\right)^{b, e}\end{array}$ \\
\hline \multirow[t]{3}{*}{1} & $\sim 115$ & 0 & 1 & -- & $\begin{array}{c}793 \pm 244 \\
(-0.06)\end{array}$ & $\begin{array}{c}2.06 \pm 0.04 \\
(-0.87)\end{array}$ \\
\hline & & 0.60 & 0.55 & $\begin{array}{l}576 \pm 84 \\
(-0.11)\end{array}$ & -- & $\begin{array}{c}2.06 \pm 0.04 \\
(-0.82)\end{array}$ \\
\hline & & 0.86 & 0.42 & $\begin{array}{c}574 \pm 56 \\
(-0.16)\end{array}$ & -- & $\begin{array}{c}2.06 \pm 0.04 \\
(-0.77)\end{array}$ \\
\hline \multirow[t]{3}{*}{6} & $\sim 31$ & 0 & 1 & -- & $\begin{array}{c}590 \pm 105 \\
(-0.08)\end{array}$ & $\begin{array}{c}1.61 \pm 0.02 \\
(-0.88)\end{array}$ \\
\hline & & 2.68 & 0.07 & $\begin{array}{c}129 \pm 4 \\
(-0.70)\end{array}$ & $\begin{array}{c}590 \pm 105 \\
(-0.18)\end{array}$ & $\begin{array}{c}1.61 \pm 0.02 \\
(-0.09)\end{array}$ \\
\hline & & 4.42 & 0.01 & $\begin{array}{l}102 \pm 2 \\
(-0.84)\end{array}$ & $\begin{array}{c}590 \pm 105 \\
(-0.11)\end{array}$ & $\begin{array}{c}1.61 \pm 0.02 \\
(-0.02)\end{array}$ \\
\hline
\end{tabular}

${ }^{a}$ Calculated from the normalized PL intensity $\left(P L / P L_{0}\right)$ of the same samples using eq 2, see the Supporting Information, Figure S10.

${ }^{b}$ Error bars are fitting errors.

${ }^{c}$ Corresponds to the charge separation process (electron transfer from QD conduction band to the LUMO of AQ). The rate of charge recombination (recombination of the electron on AQ with the hole in the QD valence band) could not be deconvolved from the component corresponding to the charge trapping process, $\tau_{2}$.

${ }^{d}$ Corresponds to an electron or hole trapping process. For QD samples with 31 bound MHA ligands, this time constant was fixed to the value extracted from the fit of the QD-only sample. For QD samples with 115 bound MHA per QD, however, $\tau_{1}$ and $\tau_{2}$ could not be deconvolved in samples with added AQ.

${ }^{e}$ Obtained from fits of the microsecond-timescale TA kinetic traces, and fixed to these values when we fit the picosecond-timescale TA kinetic traces, see Table S3B and Figure S9.

Table S3B. Time Constants for Decay of the QD Exciton for QD-AQ Complexes on Microsecond Timescale.

\begin{tabular}{ccccc}
\hline No. of Sample & $\begin{array}{c}\text { Eq. of MHA } \\
\text { bound/QD }\end{array}$ & $\begin{array}{c}\text { Eq. of AQ } \\
\text { added/QD }\end{array}$ & $\begin{array}{c}\tau_{1}(\mu \mathrm{s}) \\
\left(\mathrm{A}_{1}\right)^{a}\end{array}$ & $\begin{array}{c}\tau_{2}(\mu \mathrm{s}) \\
\left(\mathrm{A}_{2}\right)^{b}\end{array}$ \\
\hline 1 & $\sim 115$ & 0 & $2.06 \pm 0.04$ & $0.19 \pm 0.07$ \\
& & & $(-0.86)$ & $(-0.07)$ \\
& & 4000 & $1.05 \pm 0.03$ & $0.08 \pm 0.01$ \\
6 & $\sim 31$ & 0 & $(-0.73)$ & $(-0.21)$ \\
& & & $1.61 \pm 0.02$ & $0.06 \pm 0.01$ \\
& & & $(-0.83)$ & $(-0.13)$ \\
\hline
\end{tabular}

${ }^{a}$ corresponds to the radiative lifetime of $\mathrm{PbS}$ QDs

${ }^{b}$ corresponds to the surface-mediated charge trapping processes. 


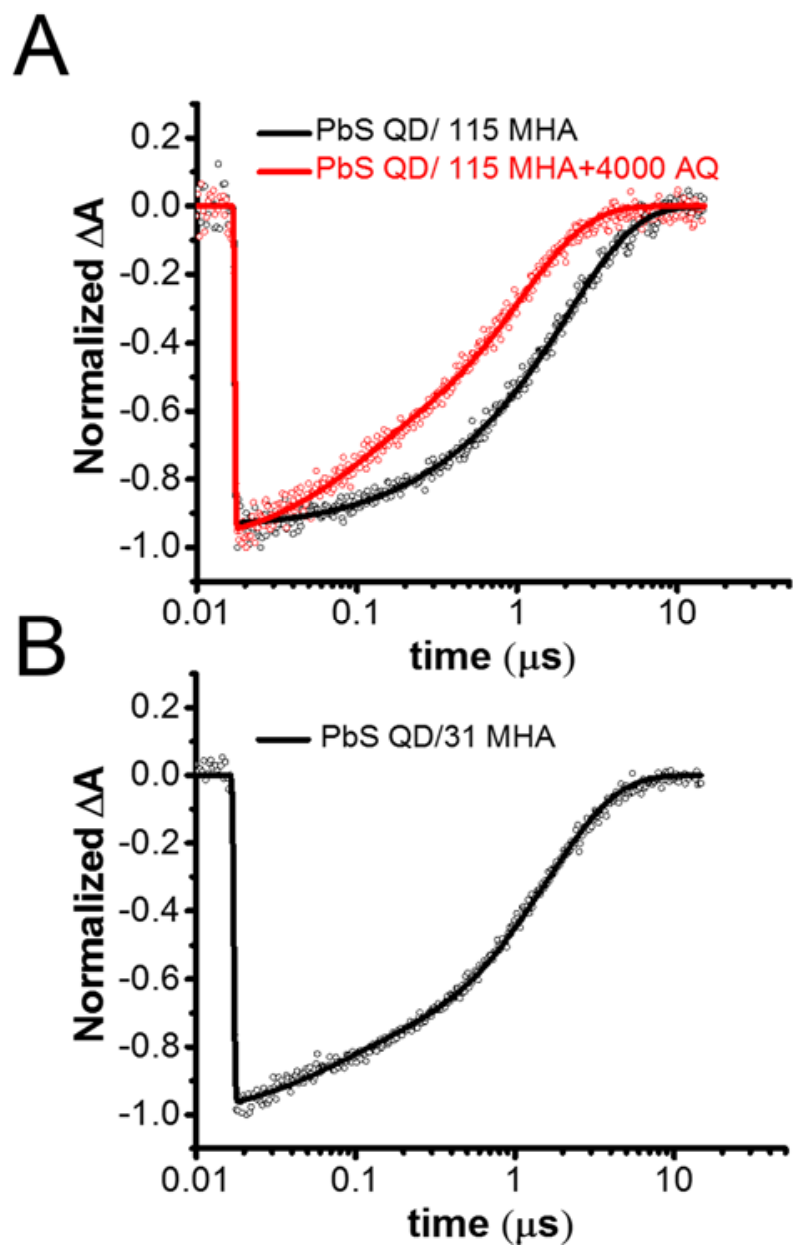

Figure S9. A) Normalized kinetic traces extracted (at $1014 \mathrm{~nm}$ ) from the TA spectrum of a $6.58 \mu \mathrm{M}$ sample of $115 \mathrm{MHA}$-capped PbS QDs mixed with 0 eq. (black) and 4000 eq. (red) of AQ. B) Normalized kinetic traces extracted (at $1039 \mathrm{~nm}$ ) from the TA spectrum of a $6.58 \mu \mathrm{M}$ sample of 31 MHA-capped PbS QDs.

We note the fact that, for 115 MHA-capped PbS QDs (sample 1) with 4000 eq of AQ added, the normalized PL intensity, $P L / P L_{0}$, and the leftover intensity of the bleach feature at the end of the 3200 ps time delay, $B / B_{0}$, are not the same due to a component of excitonic decay on the microsecond timescale, which is probably caused by dynamic quenching ${ }^{7-10}$. This decay does not show up in the microsecond kinetic traces of 31 MHA-capped QDs (Sample 6). This discrepancy does affect the accuracy of our previous calculations based on a static quenching model within pre-bound QD-AQ complexes, where we assume $P L / P L_{0}=B / B_{0}$, ie., all the eT processes completes on an ultrafast time scale and QDs with no AQ bound to their surface will undergo 
radiative recombination with a $100 \%$ yield. Instead, an additional term has to be applied to correct the discrepancy between $P L / P L_{0}$ and $B / B_{0}$, eq S3. ${ }^{7}$

$$
\frac{P L}{P L_{0}}=\frac{B}{B_{0}} \times \frac{\tau}{\tau_{0}}
$$

Here $\tau$ and $\tau_{0}$ are the reduced fluorescence lifetime for QD samples with AQs added and the original fluorescence lifetime of blank QD samples, respectively. This equation indicates that, by calculating $\lambda$ using eq 2 from the main text, we are overestimating $\lambda$ and, presumably, $K_{a}$ and $\Delta G_{o b s}$ as well. In the case of 115 MHA-capped PbS QDs with 4000 eq of AQ added, $P L / P L_{0}$ (Figure S10A, blue trace) is equal to 0.42 , while $B / B_{0}$ (Figure 3A, main text) and $\tau / \tau_{0}$ (Figure S9A, red trace) are equal to 0.88 and 0.51 , respectively. The value of $\lambda$ calculated from $B / B_{0}$ and $P L / P L_{0}$ using $\lambda=-\ln \left(B / B_{0}\right)$ and eq 2 are 0.13 and 0.86 , respectively, and we overestimate the value of $\lambda$ by 0.73 .

However, due to the small value of $\lambda$ (even after overestimation, see Table S4) that we calculated from $P L / P L_{0}$ for $115 \mathrm{MHA}$-capped QDs, we believe that this error will not strongly skew our interpretation of the data and the general trend of our results. This error may also slightly affect some other samples in the high charge density regime of QDs, such as sample 2 or 3, but we believe that this influence should be small enough to be neglected. For the sake of consistency among all our measurements, we decide to acknowledge this discrepancy and keep using eq $\mathbf{2}$ in the main text for all the calculations, while indicating $\lambda$ (in the case of 115 MHA-capped QDs only) as the upper limit, see the open symbols in Figure $\mathbf{4 B}$, main text.

Table S4. Calculated $\lambda$ for 115 MHA-capped PbS QDs Mixed with Different Amounts of AQs.

\begin{tabular}{lllllllllll}
\hline $\begin{array}{l}\text { Eq. of } \\
\text { added }\end{array}$ & AQ & & & & & & & & & \\
& 0 & 200 & 500 & 1000 & 1500 & 2000 & 3000 & 4000 & 5000 & 6000 \\
\hline$\lambda$ calculated & 0.00 & 0.10 & 0.20 & 0.31 & 0.45 & 0.49 & 0.70 & 0.77 & 1.04 & 1.14 \\
\hline
\end{tabular}


Figure S10A and S10B show the PL spectra taken under the same concentration of PbS QDs and AQs with the same 850-nm excitation wavelength as we used for ultrafast TA experiments.
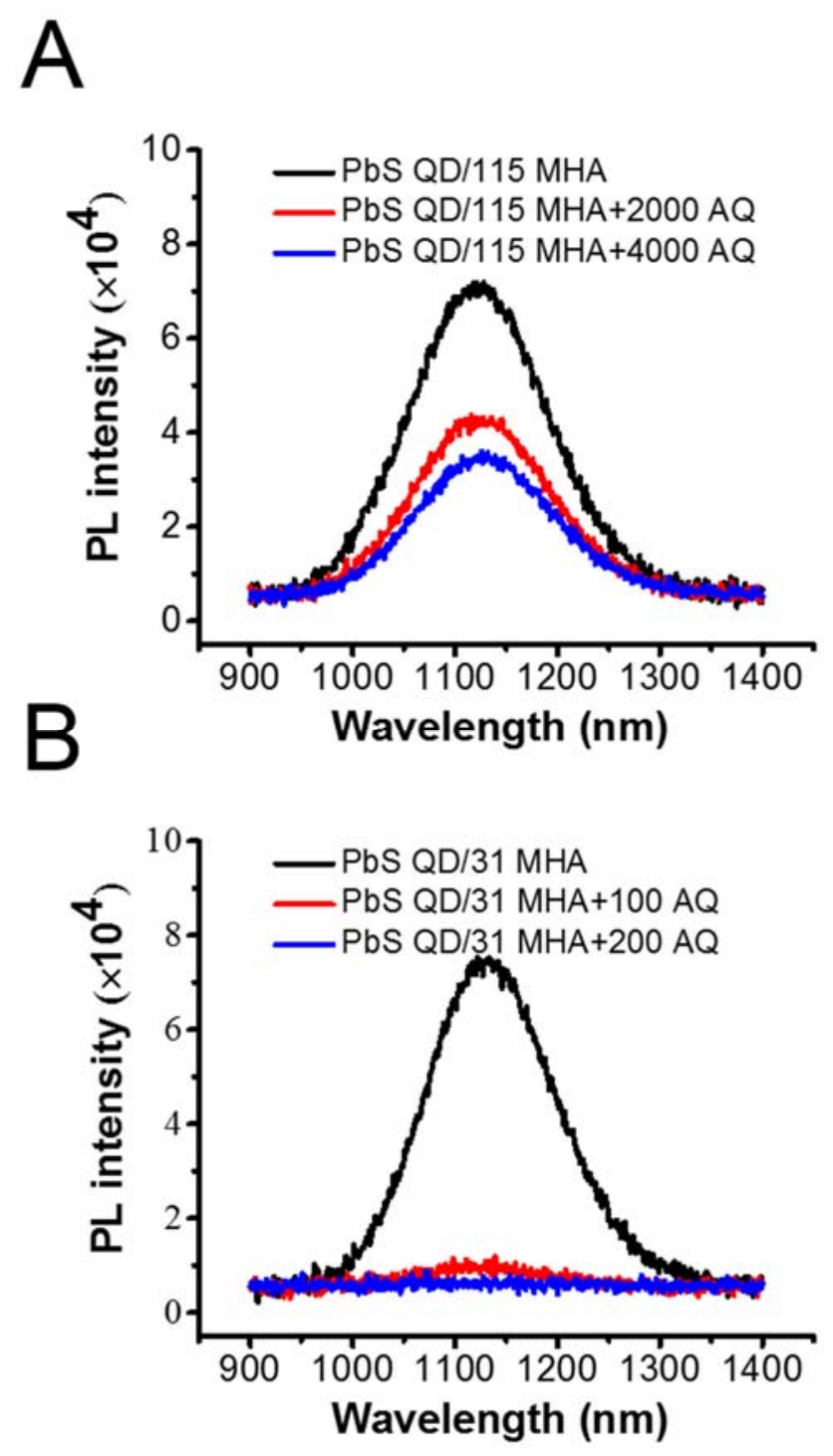

Figure S10. A) PL spectra for a $6.58 \mu \mathrm{M}$ sample of $115 \mathrm{MHA}$-capped PbS QDs with 0 (black), 2000 (red) and 4000 eq. (blue) of AQ added, respectively. B) PL spectra for a $6.58 \mu \mathrm{M}$ sample of 31 MHA-capped PbS QDs with 0 (black), 100 (red) and 200 eq. (blue) of AQ added, respectively. 
Figure S11 contains a plot of the overall rate constant for eT, $k_{o b s}$, as a function of the average number of bound AQs per PbS QD, $\lambda$, which we translate from Table S3A. Fitting these data with $k_{\text {obs }}=k_{\text {int }} \times \lambda$ yields an intrinsic eT rate constant of $2.4 \pm 0.2 \times 10^{9} \mathrm{~s}^{-1}$. The method for propagating errors in $k_{\text {obs }}$ from the errors in time constants we listed in Table S3A is also described this document, see eq S11.

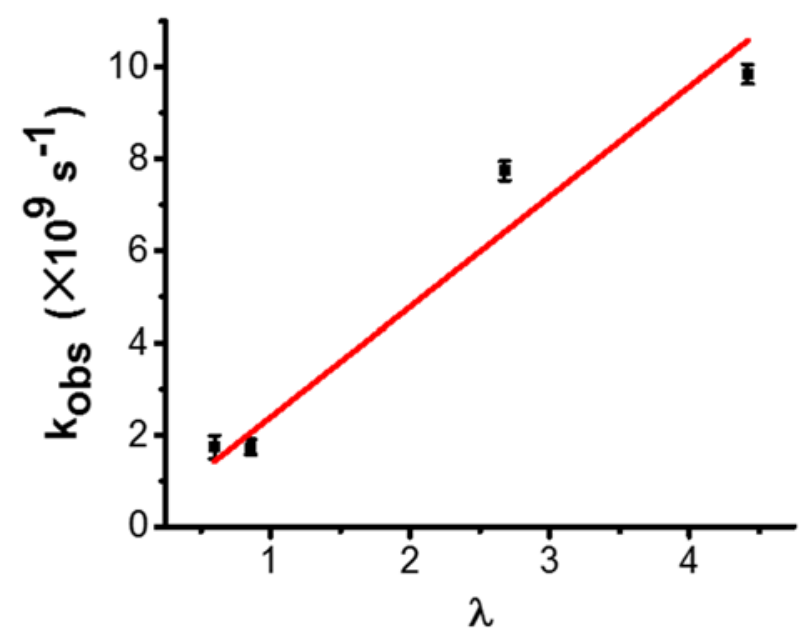

Figure S11. Plot of the overall rate constant of eT, $k_{o b s}$ as a function of bound AQs per PbS $\mathrm{QD}, \lambda$. The red line is the fitting result from a linear function after we fix the intercept to be 0 .

Derivation of Equation 6 Using the Concept of Electrochemical Equilibrium. ${ }^{11}$ With the presence of charges and local electric field at the QD/solvent interface, the total energy of an AQ that permeates through this potential barrier will be a sum of electrostatic and non-electrostatic contributions, which we could describe using eq $\mathbf{S 4}$,

$$
\mu_{e c}=\mu_{e}+\mu_{c}
$$

where $\mu_{e c}, \mu_{e}$ and $\mu_{c}$ are defined as electrochemical, electrostatic, and chemical potentials of AQ, respectively. When the system has reached electrochemical equilibrium, the electrochemical potential should be the same for AQs located anywhere in the solution. Therefore, the electrochemical potential change, $\Delta \mu_{e c}$, for an AQ to transfer from the bulk solution $(r \rightarrow \infty)$ to the proximity of a QD $\left(r \rightarrow r_{0}\right)$ could be described using eq S5, and should be zero. The term of

$$
\Delta \mu_{e c}=\Delta \mu_{e}+\Delta \mu_{c}=0
$$

electrostatic potential change, $\Delta \mu_{e}$, could be defined as the additional potential energy gained by a singly-charged AQ when it moves from the bulk solution $(r \rightarrow \infty)$ to the proximity of a QD $(r \rightarrow$ $r_{0}$ ), eq S6, while the term of chemical potential change, $\Delta \mu_{c}$, should arise from the diluted 


$$
\Delta \mu_{e}=N_{A} \times \mathrm{e}\left(\varphi\left(r_{0}\right)-\varphi(\infty)\right)=N_{A} e \varphi\left(r_{0}\right)
$$

concentration of AQ (at $r \rightarrow r_{0}$ ) relative to its concentration in the bulk solution, eq S7.

$$
\Delta \mu_{c}=\left[\mu^{\circ}+R T \ln \left(\alpha_{r_{0}}\right)\right]-\left[\mu^{\circ}+R T \ln \left(\alpha_{\infty}\right)\right]=R T \ln \left(\frac{\alpha_{r_{0}}}{\alpha_{\infty}}\right)
$$

In eq $\mathbf{S 7}, \mu^{\circ}$ is the standard chemical potential for $\mathrm{AQ}$ in aqueous solution, and $\alpha$ is the activity of AQ located at $r_{0} /$ in bulk solution. Suppose that the activity coefficient of AQ is a constant, then

$\frac{\alpha_{r_{0}}}{\alpha_{\infty}}=\frac{n_{r_{0}}}{n_{\infty}}$ holds, where $n$ is the concentration of AQ at different locations in solution. Combining this approximation with eqs S4-S7, we should be able to obtain eq S8:

$$
\begin{gathered}
N_{A} e \varphi\left(r_{0}\right)+R T \ln \left(\frac{n_{r_{0}}}{n_{\infty}}\right)=0 \\
n\left(r_{0}\right)=n(\infty) \exp \left(\frac{-N_{A} e \varphi\left(r_{0}\right)}{R T}\right)
\end{gathered}
$$

Replacing $r_{0}$ with a general variable, $r$, we therefore prove that the distribution of AQs as a function of its position comforms a Boltzmann distribution, as we described using eq $\mathbf{6}$ in the main text.

Calculations of Experimental Errors in ${ }^{1} \mathbf{H}$ NMR Measurements. In order to determine the accuracy of ${ }^{1} \mathrm{H}$ NMR measurements, we performed a series of calibration experiments (sample ag) with MHA under similar concentrations to our NMR samples used for ligand shell quantification. The concentration of MHA is therefore expressed in the unit of equivalents per QD (eq.). Figure S12 is a plot of MHA concentration measured (by integrating the triplet centered at $2.03 \mathrm{ppm}$ using 800 eq. sodium formate as an internal integration standard) as a function of the concentrations of MHA that we calculated when preparing these samples. Detailed data are listed in Table S5. We calculated the average deviation (by percentage) between the concentration of MHA we measured and the concentration calculated to be $8.26 \%$, and we use this number to further propagate the errors in our ligand shell quantification by multiplying it with the average number of MHA per QD (Table 1, main text), see eq S9. Errors we calculated for each measurement are listed in Table 1 and applied in Figure 4B in the main text. 


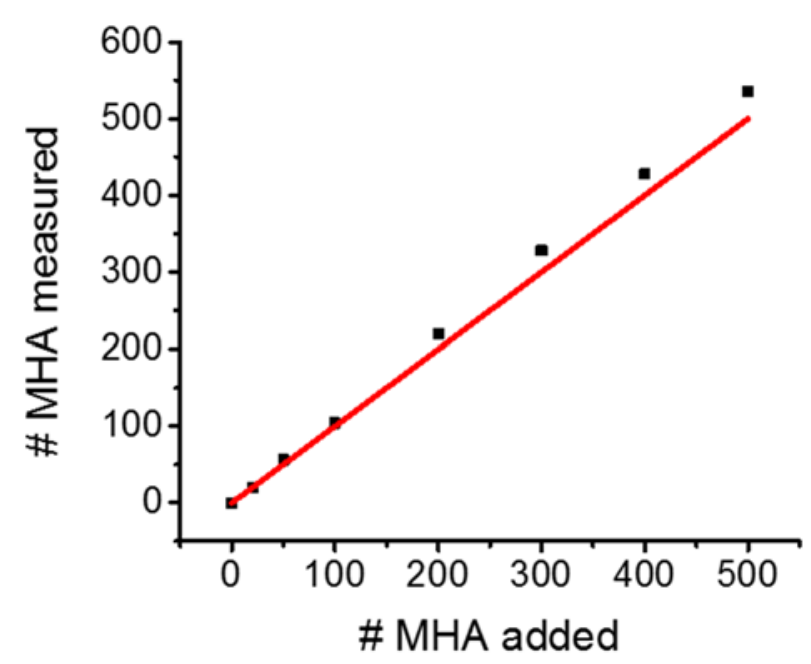

Figure S12. MHA ${ }^{1} \mathrm{H}$ NMR concentration calibration plot (sample a to $g$ ). The red line is $\mathrm{y}=\mathrm{x}$ and serves as a guide for the eye.

Table S5. Calculation of Deviation in ${ }^{1} \mathrm{H}$ NMR Calibration Experiments for MHA.

\begin{tabular}{ccccccccc}
\hline No. sample & $\mathrm{a}$ & $\mathrm{b}$ & $\mathrm{c}$ & $\mathrm{d}$ & $\mathrm{e}$ & $\mathrm{f}$ & $\mathrm{g}$ & Average \\
\hline Eq. of MHA calculated & 20 & 50 & 100 & 200 & 300 & 400 & 500 & -- \\
\hline Eq. of MHA measured & 20 & 56 & 104 & 220 & 328 & 428 & 536 & -- \\
\hline Percentage of deviation & 0.00 & 12.00 & 4.00 & 10.00 & 9.33 & 7.00 & 7.20 & 8.26 \\
\hline$\Delta(M H A)= \pm$ No. of $M H A / Q D \times 8.26 \%$ & & & $(\mathrm{~S} 9)$
\end{tabular}

Propagation of Errors in $\Delta \boldsymbol{G}_{\boldsymbol{o b s}}$. We propagated the errors of $\Delta G_{o b s}$ from the errors in $K_{a}$ that we obtained from global fitting (Figure 3A in the main text) using the equations below ${ }^{12}$ :

$$
\begin{gathered}
\Delta G_{o b s}=-R T \ln K^{\circ} \\
\Delta\left(\Delta G_{o b s}\right)=\frac{d\left(\Delta G_{o b s}\right)}{d K^{\circ}} \times \Delta K^{\circ}=-R T \times \frac{\Delta K^{\circ}}{K^{\circ}}
\end{gathered}
$$

The errors are listed in Table $\mathbf{2}$ in the main text.

Propagation of Errors in $\boldsymbol{k}_{\boldsymbol{o b s}}$. We propagated the errors of $k_{o b s}$ (see error bars in Figure S11) from the errors in $\tau_{e T}$, which we obtained from fitting the TA kinetic traces (Table S3A), using the equations below:

$$
\begin{gathered}
k_{o b s}=\frac{1}{\tau_{e T}} \\
\Delta k_{o b s}=\left|\frac{d k_{o b s}}{d \tau_{e T}}\right| \times \Delta \tau_{e T}=\left|\frac{1}{\tau_{e T}^{2}}\right| \times \Delta \tau_{e T}
\end{gathered}
$$


Conversion of No. of bound MHA to Interfacial Charge Density. Assuming that the charged MHA ligands are evenly distributed on the surface of a QD and the ligand shell is, therefore, spherically symmetric, we apply eq S12 to convert the average number of bound MHA to interfacial charge density (charges $\left./ \mathrm{nm}^{2}\right)$ :

$$
\begin{aligned}
& \text { interfacial charge density }\left(\text { charges } / \mathrm{nm}^{2}\right) \\
= & \frac{\text { No. of bound } M H A \text { per } Q D}{\text { total surface area of a charged ligand shell }}
\end{aligned}
$$

The total surface area of a charged QD ligand shell can be calculated using eq S13, where

$$
\begin{aligned}
& \text { total surface area } \\
= & 4 \times \pi \times[r(Q D)+d(M H A)]^{2}
\end{aligned}
$$

$r(Q D)=1.6 \mathrm{~nm}$ is the radius of the inorganic core of a $\mathrm{QD}$, and $d(M H A)=1.3 \mathrm{~nm}^{13}$ is the reported length of the MHA ligand. The calculated interfacial charge densities as well as the representative yields of eT upon mixing a $2.63 \mu \mathrm{M}$ QD sample with 200 eq. of AQ are listed in Table S6.

Table S6. Conversion of No. of Bound MHA to Interfacial Charge Density and the Correponding Yield of eT upon Adding 200 eq. of AQ to a $2.63 \mu M$ QD Sample.

\begin{tabular}{cccc}
\hline No. of Sample & $\begin{array}{c}\text { No. of bound MHA } \\
\text { per QD }\end{array}$ & $\begin{array}{c}\text { Interfacial Charge } \\
\text { Density } \\
\text { (charges/nm }\end{array}$ & $\begin{array}{c}\text { Yield of eT upon } \\
\text { Adding 200 Eq. of } \\
\text { AQ }^{b}\end{array}$ \\
\hline 1 & $115 \pm 9$ & 1.09 & 9.5 \\
2 & $100 \pm 8$ & 0.95 & 6.1 \\
3 & $94 \pm 8$ & 0.89 & 12.0 \\
4 & $81 \pm 7$ & 0.76 & 16.8 \\
5 & $67 \pm 6$ & 0.63 & 50.7 \\
6 & $31 \pm 3$ & 0.29 & 97.6
\end{tabular}

${ }^{a}$ Equal to the average number of bound MHA ligands per QD listed in Table 1.

${ }^{b}$ Calculated using eqs S12 and S13.

${ }^{c}$ Extracted from Figure 4B. The lower yield of eT for sample 2 compared to sample 1 (presumably caused by experimental error) is against the general trend of our data, but this discrepancy between sample 1 and 2 no longer shows up as we increase the concentration of AQ.

\section{REFERENCES}

1. $\quad$ Hines, M. A.; Scholes, G. D., Adv. Mater. 2003, 15, 1844-1849.

2. Moreels, I.; Lambert, K.; Smeets, D.; De Muynck, D.; Nollet, T.; Martins, J. C.; Vanhaecke, F.; Vantomme, A.; Delerue, C.; Allan, G.; Hens, Z., ACS Nano 2009, 3, 3023-3030.

3. Rasband, W. S. Imagej, U. S. National Institutes of Health: Bethesda, Maryland, USA, 1997-2015.

4. Bard, A. J., Electrochemical Methods : Fundamentals and Applications / Allen J. Bard, Larry R. Faulkner, 2 ed.; Wiley: New York, 2001.

5. $\quad$ Fritzinger, B.; Moreels, I.; Lommens, P.; Koole, R.; Hens, Z.; Martins, J. C., J. Am. Chem. Soc. 2009, 131, 3024-3032. 
6. Bakhmutov, V. I., Practical NMR Relaxation for Chemists; John Wiley \& Sons, Ltd.: Chichester, U.K., 2004.

7. $\quad$ Knowles, K. E.; Malicki, M.; Weiss, E. A., J. Am. Chem. Soc. 2012, 134, 12470-3.

8. Knowles, K. E.; Tagliazucchi, M.; Malicki, M.; Swenson, N. K.; Weiss, E. A., J. Phys. Chem. C 2013, 117, 15849-15857.

9. Weinberg, D. J.; He, C.; Weiss, E. A., J. Am. Chem. Soc. 2016, 138, 2319-26.

10. Merkl, J.-P.; Wolter, C.; Flessau, S.; Schmidtke, C.; Ostermann, J.; Feld, A.; Mews, A.; Weller, H., Nanoscale 2016, 8, 7402-7407.

11. Walker, D. A.; Kowalczyk, B.; de la Cruz, M. O.; Grzybowski, B. A., Nanoscale 2011, 3, 1316-1344.

12. Taylor, J. R., An Introduction to Error Analysis : The Study of Uncertainties in Physical Measurements / John R. Taylor, 2 ed.; University Science Books: Sausalito, Calif., 1997.

13. Bain, C. D.; Troughton, E. B.; Tao, Y. T.; Evall, J.; Whitesides, G. M.; Nuzzo, R. G., J. Am. Chem. Soc. 1989, 111, 321-335. 